\title{
TOWERS OF NODAL BUBBLES FOR THE BAHRI-CORON PROBLEM IN PUNCTURED DOMAINS
}

\author{
MÓNICA CLAPP, JORGE FAYA, AND FILOMENA PACELLA
}

\begin{abstract}
Let $\Omega$ be a bounded smooth domain in $\mathbb{R}^{N}$ which contains a ball of radius $R$ centered at the origin, $N \geq 3$. Under suitable symmetry assumptions, for each $\delta \in(0, R)$, we establish the existence of a sequence $\left(u_{m, \delta}\right)$ of nodal solutions to the critical problem

$$
-\Delta u=|u|^{2^{*}-2} u \text { in } \Omega_{\delta}:=\{x \in \Omega:|x|>\delta\}, \quad u=0 \text { on } \partial \Omega_{\delta},
$$

where $2^{*}:=\frac{2 N}{N-2}$ is the critical Sobolev exponent. We show that, if $\Omega$ is strictly starshaped then, for each $m \in \mathbb{N}$, the solutions $u_{m, \delta}$ concentrate and blow up at 0 , as $\delta \rightarrow 0$, and their limit profile is a tower of nodal bubbles, i.e., it is a sum of rescaled nonradial sign-changing solutions to the limit problem

$$
-\Delta u=|u|^{2^{*}-2} u, \quad u \in D^{1,2}\left(\mathbb{R}^{N}\right)
$$

centered at the origin.

KEY WORDS: Semilinear elliptic boundary value problem; critical nonlinearity; nodal solutions; punctured domains; bubble-towers; asymptotic analysis.

2010 MSC: 35J61, 35B33, 35B40.
\end{abstract}

\section{INTRODUCTION}

Let $\Omega$ be a bounded smooth domain in $\mathbb{R}^{N}$ which contains a ball of radius $R>0$ centered at the origin, $N \geq 3$. Our goal is to exhibit new blow-up phenomena for solutions to the problem

$$
\begin{cases}-\Delta u=|u|^{2^{*}-2} u & \text { in } \Omega_{\delta} \\ u=0 & \text { on } \partial \Omega_{\delta}\end{cases}
$$

as $\delta \rightarrow 0$, where $2^{*}:=\frac{2 N}{N-2}$ is the critical Sobolev exponent and

$$
\Omega_{\delta}:=\{x \in \Omega:|x|>\delta\}, \quad \delta \in(0, R) .
$$

It is well known that the existence of a nontrivial solution to the problem

$$
-\Delta u=|u|^{2^{*}-2} u \text { in } \Omega, \quad u=0 \text { on } \partial \Omega,
$$

depends on the domain. Pohozhaev's identity, together with the unique continuation principle, implies that this problem does not have a nontrivial solution if $\Omega$ is

Date: March 7, 2017.

M. Clapp was partially supported by CONACYT grant 237661 (México) and PAPIIT-DGAPAUNAM grant IN104315 (Mexico). J. Faya was partially supported by FONDECYT postdoctoral grant 3150172 (Chile), Fondo Basal CMM (Chile) and Millennium Nucleus Center for Analysis of PDE NC130017 (Chile). F. Pacella was partially supported by exchange funds of the Università "Sapienza" of Roma, and GNAMPA-INDAM funds (Italy). 
strictly starshaped. The first nontrivial result was obtained by Coron, who showed in [12] that the problem (1.1) has at least one positive solution for $\delta$ small enough. A few years later, a mayor breakthrough was achieved by Bahri and Coron who established the existence of a positive solution in every domain whose homology with $\mathbb{Z}_{2}$-coefficients is nontrivial. Problem (1.2) is now refered to as the Bahri-Coron problem.

Multiplicity of solutions to the problem (1.1), for $\delta$ small, has been exhibited, e.g., in $[8,9,11]$ and some of the references therein. Multiplicity results are also available for more general domains with nontrivial topology under suitable symmetry assumptions; see [6, 9].

Ge, Musso and Pistoia proved in [15] that the number of sign-changing solutions to the problem (1.1) becomes arbitrarily large as $\delta$ goes to zero. The limit profile of their solutions is given by

$$
\sum_{j=1}^{\ell}(-1)^{j} \varepsilon_{j, \delta}^{(2-N) / 2} U\left(\frac{\cdot}{\varepsilon_{j, \delta}}\right),
$$

where $U$ is the so-called standard bubble, which is the only positive solution to the problem

$$
-\Delta u=|u|^{2^{*}-2} u, \quad u \in D^{1,2}\left(\mathbb{R}^{N}\right),
$$

up to sign, translation and rescaling. The rescaling parameters $\varepsilon_{j, \delta}$ tend to 0 with suitable velocities, as $\delta \rightarrow 0$. These solutions are called bubble-towers.

It was recently shown in [5] that there are nonradial sign-changing solutions to the problem (1.4) which occur as limit profiles in concentration phenomena. We shall call these solutions $\phi$-nodal bubbles. Their precise definition is given below. Further, it was shown in [10] that, under some symmetry assumptions, least energy $\phi$-nodal bubbles occur as the limit profile of sign-changing solutions to the problem (1.1), as $\delta \rightarrow 0$.

It is natural to ask whether there are solutions to (1.1) whose limit profile is a tower of $\phi$-nodal bubbles, i.e., a sum of the form (1.3) with $U$ replaced by a $\phi$-nodal bubble. A general answer is not easy. The Ljapunov-Schmidt reduction method, which was used in [15] to produce bubble-towers, cannot be applied to $\phi$-nodal bubbles, since nothing is known about the spectrum of the linearized operator at these bubbles.

In this paper we will establish the existence of towers of $\phi$-nodal bubbles in domains with particular symmetries, using variational methods. Next, we present and discuss our main result.

Let $\Gamma$ be a closed subgroup of the group $O(N)$ of linear isometries of $\mathbb{R}^{N}$ and $\phi: \Gamma \rightarrow \mathbb{Z}_{2}:=\{1,-1\}$ be a continuous homomorphism of groups. Recall that the $\Gamma$-orbit of a point $x \in \mathbb{R}^{N}$ is the set $\Gamma x:=\{\gamma x: \gamma \in \Gamma\}$. We assume that 
$\left(\mathbf{S}_{1}\right) \operatorname{dim} \Gamma x>0$ for every $x \in \mathbb{R}^{N} \backslash\{0\}$,

$\left(\mathbf{S}_{2}\right)$ there exists $\xi \in \mathbb{R}^{N}$ such that $\{\gamma \in \Gamma: \gamma \xi=\xi\} \subset \operatorname{ker} \phi$.

A function $u: \mathbb{R}^{N} \rightarrow \mathbb{R}$ is said to be $\phi$-equivariant if it satisfies

$$
u(\gamma x)=\phi(\gamma) u(x) \quad \text { for all } \gamma \in \Gamma, x \in \mathbb{R}^{N} .
$$

Note that, if $\phi$ is surjective, then every nontrivial $\phi$-equivariant function is nonradial and changes sign. If $\phi$ is surjective, any nontrivial $\phi$-equivariant solution to the limit problem (1.4) will be called a $\phi$-nodal bubble.

For $u \in D^{1,2}\left(\mathbb{R}^{N}\right)$ we set

$$
\|u\|:=\left(\int_{\mathbb{R}^{N}}|\nabla u|^{2}\right)^{1 / 2}
$$

We will prove the following result.

Theorem 1.1. If $\Omega$ is $\Gamma$-invariant then, for each $\delta \in(0, R)$ and each positive integer $m$, the problem (1.1) has a $\phi$-equivariant solution $u_{m, \delta}$, and there exists a positive constant $C_{N}$, which depends only on $N$, such that

$$
C_{N} m \leq\left\|u_{m, \delta}\right\|^{2} \quad \text { for every } \delta \in(0, R), m \in \mathbb{N} .
$$

If, moreover, $\Omega$ is strictly starshaped, then, for every positive integer $m$ and every sequence $\left(\delta_{k}\right)$ in $(0, R)$ with $\delta_{k} \rightarrow 0$, after passing to a subsequence, there exist a a positive integer $\ell$ and, for each $j=1, \ldots, \ell$, a nontrivial $\phi$-equivariant solution $\omega_{j}$ to the limit problem $(1.4)$ and a sequence $\left(\varepsilon_{j, k}\right)$ in $(0, \infty)$ such that

(i) $\varepsilon_{j, k} \rightarrow 0$ and $\frac{\varepsilon_{j, k}}{\delta_{k}} \rightarrow \infty$ as $k \rightarrow \infty$, for each $j=1, \ldots, \ell$;

(ii) $\left\|u_{m, \delta_{k}}-\sum_{j=1}^{\ell} \varepsilon_{j, k}^{(2-N) / 2} \omega_{j}\left(\frac{\cdot}{\varepsilon_{j, k}}\right)\right\| \rightarrow 0$ as $k \rightarrow \infty$;

(iii) $\lim _{k \rightarrow \infty}\left\|u_{m, \delta_{k}}\right\|^{2}=\sum_{j=1}^{\ell}\left\|\omega_{j}\right\|^{2}$.

In particular, Theorem 1.1 says that, if $\Omega$ is $\Gamma$-invariant and strictly starshaped and $\phi$ is surjective, then, for each $m \in \mathbb{N}$, there is a sequence $\delta_{k} \rightarrow 0$ and a solution $u_{m, \delta_{k}}$ to the problem (1.1) in $\Omega_{\delta_{k}}$ whose limit profile is a tower

$$
\sum_{j=1}^{\ell} \varepsilon_{j, k}^{(2-N) / 2} \omega_{j}\left(\frac{\cdot}{\varepsilon_{j, k}}\right)
$$

of $\ell \phi$-nodal bubbles $\omega_{1}, \ldots, \omega_{\ell}$. Moreover, as

$$
C_{N} m \leq \lim _{k \rightarrow \infty}\left\|u_{m, \delta_{k}}\right\|^{2}=\sum_{j=1}^{\ell}\left\|\omega_{j}\right\|^{2}
$$

our theorem yields the following alternative: either the set of $\phi$-nodal bubbles is unbounded in $D^{1,2}\left(\mathbb{R}^{N}\right)$, or, for arbitrarily large $\ell$, there are solutions to the problem (1.1) whose limit profile is a tower of $\ell \phi$-nodal bubbles. 
In the special case when $\Omega$ is a ball and, hence, $\Omega_{\delta}$ is an annulus, one can use the geometry of the problem to produce a tower of $\ell \phi$-nodal bubbles for every $\ell \in \mathbb{N}$. Moreover, each $\phi$-nodal bubble in the tower has minimal energy. This will be proved in Section 4 .

It is not always true that the set of $\phi$-equivariant solutions to the limit problem (1.4) is unbounded in $D^{1,2}\left(\mathbb{R}^{N}\right)$. For instance, if $\Gamma=O(N)$ and $\phi \equiv 1$, then a $\phi$-equivariant function is simply a radial function and the standard bubble is the only radial solution to this problem, up to sign and dilations. Of course, $\phi$ is not surjective in this case.

On the other hand, W. Ding showed that the problem (1.4) has an unbounded sequence of sign-changing solutions which are invariant under the conformal action of the group $\mathcal{G}:=O(k) \times O(m)$ on $\mathbb{R}^{N}$, induced by the orthogonal action of $\mathcal{G}$ on the $N$-dimensional sphere $\mathbb{S}^{N}$ via the stereographic projection, if $k+m=N+1$, $m, k \geq 2$ [14]. This action is very different from the linear actions we are considering here: the $\mathcal{G}$-orbit of every point of $\mathbb{R}^{N}$ has positive dimension, so the Sobolev embedding, when restricted to the space of $\mathcal{G}$-invariant functions, turns out to be compact, and standard variational methods yield a sequence of sign-changing solutions which is unbounded in $D^{1,2}\left(\mathbb{R}^{N}\right)$. In contrast, for the standard linear action of $O(N)$, the $O(N)$-orbit of the origin consists of that single point. This allows blow-up, and explains why standard bubbles and $\phi$-nodal bubbles occur in concentration phenomena.

It is an interesting open question to determine whether, for surjective $\phi$, the set of $\phi$-nodal bubbles is unbounded in $D^{1,2}\left(\mathbb{R}^{N}\right)$ or not.

Let us give an example of the kind of symmetries we are considering. For $N \geq 4$ we write the points in $\mathbb{R}^{N} \equiv \mathbb{C}^{2} \times \mathbb{R}^{N-4}$ as $(z, y)$ with $z=\left(z_{1}, z_{2}\right) \in \mathbb{C}^{2}, y \in \mathbb{R}^{N-4}$, and we consider the group $\Gamma$ generated by $\left\{\mathrm{e}^{\mathrm{i} \vartheta}, \alpha, \tau: \vartheta \in[0,2 \pi), \alpha \in O(N-4)\right\}$ acting on a point $(z, y)$ by

$$
\mathrm{e}^{\mathrm{i} \vartheta}(z, y):=\left(\mathrm{e}^{\mathrm{i} \vartheta} z, y\right), \quad \alpha(z, y):=(z, \alpha y), \quad \tau\left(z_{1}, z_{2}, y\right):=\left(-\bar{z}_{2}, \bar{z}_{1}, y\right) .
$$

The homomorphism $\phi: \Gamma \rightarrow \mathbb{Z}_{2}$ is given by $\phi\left(\mathrm{e}^{\mathrm{i} \vartheta}\right):=1, \phi(\alpha):=1, \phi(\tau):=-1$. So $\phi$ is surjective. If $N=4$ the $\Gamma$-orbit of each point in $\mathbb{R}^{4} \backslash\{0\}$ is homeomorphic to the disjoint union $\mathbb{S}^{1} \sqcup \mathbb{S}^{1}$ of two circles, whereas for $N \geq 5$ and $(z, y) \in \mathbb{R}^{N} \backslash\{0\}$,

$$
\Gamma(z, y) \cong \begin{cases}\mathbb{S}^{N-5} & \text { if } z=0 \text { and } y \neq 0, \\ \mathbb{S}^{1} \sqcup \mathbb{S}^{1} & \text { if } z \neq 0 \text { and } y=0, \\ \left(\mathbb{S}^{1} \times \mathbb{S}^{N-5}\right) \sqcup\left(\mathbb{S}^{1} \times \mathbb{S}^{N-5}\right) & \text { if } z \neq 0 \text { and } y \neq 0 .\end{cases}
$$

Hence, assumption $\left(\mathbf{S}_{1}\right)$ holds true if $N=4$ or $N \geq 6$. Notice that $\left(\mathbf{S}_{2}\right)$ is satisfied by any point $\xi=(z, y)$ with $z \neq 0$. A function $u$ is $\phi$-equivariant if

$$
u(z, y)=u\left(\mathrm{e}^{\mathrm{i} \vartheta} z, \alpha y\right) \quad \text { and } \quad u\left(z_{1}, z_{2}, y\right)=-u\left(-\bar{z}_{2}, \bar{z}_{1}, y\right),
$$


for all $\vartheta \in[0,2 \pi), \alpha \in O(N-4),(z, y) \in \mathbb{R}^{N}$. Thus, if $z \neq 0$, the function $u$ takes some constant value $c$ on one of the components of $\Gamma(z, y)$ and the value $-c$ on the other component.

We wish to say a few words about the proof of Theorem 1.1. Assumption $\left(\mathbf{S}_{1}\right)$ implies that every $\Gamma$-orbit in $\Omega_{\delta}$ has positive dimension. Hence, Theorem 6.1 in [5] yields an unbounded sequence of $\phi$-equivariant solutions to the problem (1.1), for each $\delta \in(0, R)$. The new piece of information is the estimate (1.6) for the growth of the norm of these solutions. We obtain this estimate following the ideas introduced by Bahri and Lions in [2]. A finer estimate, which takes the symmetries into account, was recently obtained in [1] for more general exponents, but the constant involved depends on the domain. We stress that the constant $C_{N}$ given here depends only on $N$.

To obtain the limit profile of the solutions, as $\delta \rightarrow 0$, we use concentrationcompactness arguments. Struwe used this kind of arguments in [20] to describe the Palais-Smale sequences of the energy functional associated to the Bahri-Coron problem. A priori it is not clear whether, for fixed $m \in \mathbb{N}$ and $\delta_{k} \rightarrow 0$, the solutions $u_{m, \delta_{k}}$ to the problem (1.1) in $\Omega_{\delta_{k}}$ form a Palais-Smale sequence for the Bahri-Coron problem in the whole domain $\Omega$. But they do form a concentrating sequence in the sense of Definition 3.3, given below. In contrast to Struwe's argument, where the nonexistence of solutions in a half-space plays an important role, the argument that we give to describe concentrating sequences uses the fact that there are no solutions to the Bahri-Coron problem in the complement of a ball; see Proposition 3.5 below.

This paper is organized as follows: in Section 2 we estimate the growth of the minimax values for the functional associated to the Bahri-Coron problem in symmetric domains with positive dimensional orbits. Section 3 is devoted to the description of concentrating sequences and to the proof of Theorem 1.1. In Section 4 we consider the special case when $\Omega$ is a ball. We establish the existence of towers of $\ell \phi$-nodal bubbles for every $\ell \in \mathbb{N}$ and give a precise description of them.

\section{The growth of Symmetric minimax Values at the CRitical exponent}

Let $\Gamma$ be a closed subgroup of $O(N)$ and $\phi: \Gamma \rightarrow \mathbb{Z}_{2}$ be a continuous homomorphism of groups. Recall that a function $u: \mathbb{R}^{N} \rightarrow \mathbb{R}$ is said to be $\phi$-equivariant if it satisfies (1.5).

Let $\Theta$ be a $\Gamma$-invariant bounded smooth domain in $\mathbb{R}^{N}$ such that

$$
\left\{\gamma \in \Gamma: \gamma x_{0}=x_{0}\right\} \subset \operatorname{ker} \phi \quad \text { for some } x_{0} \in \Theta .
$$

This property guarantees that the space

$$
D_{0}^{1,2}(\Theta)^{\phi}:=\left\{u \in D_{0}^{1,2}(\Theta): u \text { is } \phi \text {-equivariant }\right\}
$$


is infinite dimensional; cf. [3]. As usual, $D_{0}^{1,2}(\Theta)$ denotes the closure of $\mathcal{C}_{c}^{\infty}(\Theta)$ in the Hilbert space $D^{1,2}\left(\mathbb{R}^{N}\right):=\left\{u \in L^{2^{*}}\left(\mathbb{R}^{N}\right): \nabla u \in L^{2}\left(\mathbb{R}^{N}, \mathbb{R}^{N}\right)\right\}$ with the norm

$$
\|u\|:=\left(\int_{\mathbb{R}^{N}}|\nabla u|^{2}\right)^{1 / 2}
$$

We consider the problem

$$
\begin{cases}-\Delta u=|u|^{2^{*}-2} u & \text { in } \Theta \\ u=0 & \text { on } \partial \Theta, \\ u(\gamma x)=\phi(\gamma) u(x) & \forall \gamma \in \Gamma, x \in \Theta .\end{cases}
$$

By Palais' principle of symmetric criticality [19], the solutions to this problem are the critical points of the functional $J_{\Theta}: D_{0}^{1,2}(\Theta)^{\phi} \rightarrow \mathbb{R}$ given by

$$
J_{\Theta}(u):=\frac{1}{2}\|u\|^{2}-\frac{1}{2^{*}}|u|_{2^{*}}^{2^{*}},
$$

where $|u|_{2^{*}}^{2^{*}}:=\int_{\mathbb{R}^{N}}|u|^{2^{*}}$. The nontrivial solutions lie on the Nehari manifold

$$
\mathcal{N}_{\Theta}^{\phi}:=\left\{u \in D_{0}^{1,2}(\Theta)^{\phi}: u \neq 0,\|u\|^{2}=|u|_{2^{*}}^{2^{*}}\right\},
$$

which is of class $\mathcal{C}^{2}$, radially diffeomorphic to the unit sphere in $D_{0}^{1,2}(\Theta)^{\phi}$, and a natural constraint for $J_{\Theta}$.

For each $c \in \mathbb{R}$, set $J_{\Theta}^{c}:=\left\{u \in \mathcal{N}_{\Theta}^{\phi}: J_{\Theta}(u) \leq c\right\}$. Note that $J_{\Theta}^{c}$ is symmetric with respect to the origin, i.e., $u \in J_{\Theta}^{c}$ iff $-u \in J_{\Theta}^{c}$, and that $0 \notin J_{\Theta}^{c}$. For each $m \in \mathbb{N}$ we define

$$
c_{m, \Theta}^{\phi}:=\inf \left\{c \in \mathbb{R}: \operatorname{genus}\left(J_{\Theta}^{c}\right) \geq m\right\},
$$

where genus $(X)$ is the Krasnoselskii genus of $X$. Recall that, if $X \subset D^{1,2}\left(\mathbb{R}^{N}\right)$ is nonempty and symmetric with respect to the origin, then $\operatorname{genus}(X)$ is the smallest number $m \in \mathbb{N}$ for which there exists a continuous map $f: X \rightarrow \mathbb{S}^{m-1}$ such that $f(-u)=-f(u)$ for every $u \in X$, where $\mathbb{S}^{m-1}$ is the unit sphere in $\mathbb{R}^{m}$. If no such map exists, we define genus $(X):=\infty$.

Theorem 2.1. Assume that the $\Gamma$-orbit $\Gamma x$ of every point $x \in \Theta$ has positive dimension. Then, $c_{m, \Theta}^{\phi}$ is a critical value of the functional $J_{\Theta}: \mathcal{N}_{\Theta}^{\phi} \rightarrow \mathbb{R}$ and there exists a positive constant $B_{N}$, which depends only on $N$, such that

$$
B_{N} m \leq c_{m, \Theta}^{\phi}
$$

for every $m \in \mathbb{N}$.

Proof. Since every $\Gamma$-orbit in $\Theta$ has positive dimension, the argument given to prove Theorem 6.1 in [5] shows that the functional $J_{\Theta}: \mathcal{N}_{\Theta}^{\phi} \rightarrow \mathbb{R}$ satisfies the Palais-Smale condition. Then, standard arguments allow to show that $c_{m, \Theta}^{\phi} \in(0, \infty)$ and that $c_{m, \Theta}^{\phi}$ is a critical value of this functional for every $m \in \mathbb{N}$; see, e.g., Chapter II in [21]. Moreover, as the Palais-Smale condition holds true, the argument used by Bahri and Lions in [2] to estimate the minimax values in the subcritical case, can also be used to prove the estimate (2.3). We sketch the proof of this estimate. 
Let $\sigma^{\phi}(u)$ be the generalized Morse index of a critical point $u$ of $J_{\Theta}: \mathcal{N}_{\Theta}^{\phi} \rightarrow$ $\mathbb{R}$, i.e., $\sigma^{\phi}(u)$ is the dimension of the eigenspace corresponding to all nonpositive eigenvalues of the operator $L_{u}:=-\Delta-\left(2^{*}-1\right)|u|^{2^{*}-2}$ acting on the tangent space $T_{u} \mathcal{N}_{\Theta}^{\phi}$ to the manifold $\mathcal{N}_{\Theta}^{\phi}$ at the point $u$. Then, as $D_{0}^{1,2}(\Theta)^{\phi}=T_{u} \mathcal{N}_{\Theta}^{\phi} \oplus \mathbb{R} u$ and $L_{u}$ is negative definite on $\mathbb{R} u$, the generalized Morse index of this operator in the space $D_{0}^{1,2}(\Theta)^{\phi}$ is $\sigma^{\phi}(u)+1$. The semiclassical inequality of Cwikel [13], Lieb [16] and Rosenbljum [19] states that there is a positive constant $A_{N}$, which depends only on $N$, such that for every nonpositive potential $V \in L^{N / 2}\left(\mathbb{R}^{N}\right)$, the generalized Morse index of the Schrödinger operator $-\Delta+V$ in $L^{2}\left(\mathbb{R}^{N}\right)$ is bounded from above by $A_{N} \int_{\mathbb{R}^{N}}|V|^{N / 2}$. Therefore, if $u \in \mathcal{N}_{\Theta}^{\phi}$ is a critical point of $J_{\Theta}$ with critical value $c_{m, \Theta}^{\phi}$, setting $B_{N}^{-1}:=A_{N} N\left(\frac{N+2}{N-2}\right)^{N / 2}$, we have that

$$
\sigma^{\phi}(u)+1 \leq A_{N}\left(2^{*}-1\right)^{N / 2} \int_{\Theta}|u|^{2^{*}}=A_{N} N\left(\frac{N+2}{N-2}\right)^{N / 2} J_{\Theta}(u)=B_{N}^{-1} c_{m, \Theta}^{\phi} .
$$

We claim that there exists a critical point $u \in \mathcal{N}_{\Theta}^{\phi}$ of $J_{\Theta}$ with critical value $c_{m, \Theta}^{\phi}$ and $\sigma^{\phi}(u) \geq m-1$. This fact, combined with inequality (2.4) immediately yields (2.3).

To prove this claim we argue by contradiction, i.e., we assume that $\sigma^{\phi}(u)<m-1$ for every critical point $u \in \mathcal{N}_{\Theta}^{\phi}$ of $J_{\Theta}$ with critical value $c_{m, \Theta}^{\phi}=: c$.

First, we consider the case when $J_{\Theta}$ has finitely many critical points $\pm u_{1}, \ldots, \pm u_{k}$ with critical value $c$ and all of them are nondegenerate. Let $\mu_{i}$ be the Morse index of $u_{i}$. Then, there is a splitting of the tangent space $T_{u_{i}} \mathcal{N}_{\Theta}^{\phi}$ as an orthogonal sum $X_{i} \oplus Y_{i}$ with $\operatorname{dim} X_{i}=\mu_{i}$ such that, in local coordinates $(x, y) \in X_{i} \oplus Y_{i}$ around $u_{i}$, $J_{\Theta}$ can be written as $(x, y) \mapsto|y|^{2}-|x|^{2}$. As $J_{\Theta}: \mathcal{N}_{\Theta}^{\phi} \rightarrow \mathbb{R}$ satisfies the Palais-Smale condition, taking small enough coordinate neighborhoods $V_{i}, U_{i}$ of $u_{i}$ with $\bar{V}_{i} \subset U_{i}$ and following the flow of the vector field $(x, y) \mapsto(0,-y)$ in $V_{i}$, the flow of $-\nabla J_{\Theta}$ outside of $U_{i}$ and the flow of a convex combination of both vector fields in $U_{i} \backslash V_{i}$, at a suitable speed, one can construct an odd deformation

$$
J_{\Theta}^{c+\varepsilon} \longrightarrow J_{\Theta}^{c-\varepsilon} \cup B^{\mu_{1}} \cup\left(-B^{\mu_{1}}\right) \cup \cdots \cup B^{\mu_{k}} \cup\left(-B^{\mu_{k}}\right),
$$

for some small enough $\varepsilon>0$, where $B^{\mu_{i}}$ is homeomorphic to a closed $\mu_{i}$-dimensional ball, the sets $B^{\mu_{1}},-B^{\mu_{1}}, \ldots, B^{\mu_{k}},-B^{\mu_{k}}$ are pairwise disjoint, and $J_{\Theta}^{c-\varepsilon} \cap B^{\mu_{i}}=$ $\partial B^{\mu_{i}}$. The definition of $c_{m, \Theta}^{\phi}$ implies that there exists an odd map $f: J_{\Theta}^{c-\varepsilon} \rightarrow$ $\mathbb{S}^{m-2}$. As $\mu_{i}<m-1$, the restriction of $f$ to $\partial B^{\mu_{i}}$ is nullhomotopic and, using a nullhomotopy for each $i=1, \ldots, k$, one can extend $f$ to an odd map

$$
J_{\Theta}^{c-\varepsilon} \cup B^{\mu_{1}} \cup\left(-B^{\mu_{1}}\right) \cup \cdots \cup B^{\mu_{k}} \cup\left(-B^{\mu_{k}}\right) \longrightarrow \mathbb{S}^{m-2}
$$


whose composition with the deformation (2.5) gives an odd map $J_{\Theta}^{c+\varepsilon} \rightarrow \mathbb{S}^{m-2}$, contradicting the fact that genus $\left(J_{\Theta}^{c+\varepsilon}\right) \geq m$. Therefore, $\mu_{i} \geq m-1$ for some $i$, as claimed.

Next, we consider the general case. Note that, as $J_{\Theta}: \mathcal{N}_{\Theta}^{\phi} \rightarrow \mathbb{R}$ satisfies the Palais-Smale condition, there are regular values $a$ and $b$ of $J_{\Theta}$ such that $a<c<b$ and the set $Z:=\left\{u \in \mathcal{N}_{\Theta}^{\phi}: J_{\Theta}^{\prime}(u)=0, J_{\Theta}(u) \in[a, b]\right\}$ is compact. Fix $0<$ $\delta<\operatorname{dist}\left(Z, \mathcal{N}_{\Theta}^{\phi} \backslash J_{\Theta}^{-1}(a, b)\right)$. By Theorem 2.2 in [17] and Theorem 5.7 in [4], there exists a $\mathcal{C}^{2}$-function $I: \mathcal{N}_{\Theta}^{\phi} \rightarrow \mathbb{R}$ which satisfies the Palais-Smale condition, such that $I(u)=J_{\Theta}(u)$ if $\operatorname{dist}(u, Z) \geq \delta$ and $I$ has finitely many critical points in $J_{\Theta}^{-1}[a, b]$; they are nondegenerate and their Morse index is smaller than $m-1$. Let $d_{1}<\cdots<d_{j}$ be the critical values of $I$ in $J_{\Theta}^{-1}[a, b]$. As $a$ and $b$ are regular values of $I$, we have that $a<d_{1}$ and $d_{m}<b$ and, since $I$ satisfies the Palais-Smale condition, there are odd maps

$$
\begin{array}{ll}
I^{d_{1}-\varepsilon} \rightarrow I^{a} & \text { for every } \varepsilon \in\left(0,\left|d_{1}-a\right|\right), \\
I^{d_{i+1}-\varepsilon} \rightarrow I^{d_{i}+\varepsilon} & \text { for every } \varepsilon \in\left(0, \frac{1}{2}\left|d_{i+1}-d_{i}\right|\right), i=2, \ldots, j, \\
I^{b} \rightarrow I^{d_{j}+\varepsilon} & \text { for every } \varepsilon \in\left(0,\left|b-d_{j}\right|\right) .
\end{array}
$$

Now, as $I^{a}=J_{\Theta}^{a}$ and $a<c=c_{m, \Theta}^{\phi}$, there is an odd map $I^{a} \rightarrow \mathbb{S}^{m-2}$. This map, composed with the first map in (2.6), yields an odd map $I^{d_{1}-\varepsilon} \rightarrow \mathbb{S}^{m-2}$ for every $\varepsilon \in\left(0,\left|d_{1}-a\right|\right)$. The argument given above allows us to extend this map to an odd map $I^{d_{1}+\varepsilon_{1}} \rightarrow \mathbb{S}^{m-2}$ for some $\varepsilon_{1}>0$. This map, composed with the map in (2.6), yields an odd map $I^{d_{2}-\varepsilon} \rightarrow \mathbb{S}^{m-2}$ for every $\varepsilon \in\left(0, \min \left\{\frac{1}{2}\left|d_{2}-d_{1}\right|, \varepsilon_{1}\right\}\right)$. Continuing in this way, we obtain an odd map $I^{b} \rightarrow \mathbb{S}^{m-2}$. But, since $I^{b}=J_{\Theta}^{b}$ and $b>c=c_{m, \Theta}^{\phi}$, we have that genus $\left(I^{b}\right) \geq m$. So this is a contradiction, and the claim is proved.

\section{Symmetries AND BlOW-UP AT A Single POINT}

Let $\Gamma$ be a closed subgroup of $O(N)$ and $\phi: \Gamma \rightarrow \mathbb{Z}_{2}$ be a continuous homomorphism of groups which satisfy the assumptions $\left(\mathbf{S}_{1}\right)$ and $\left(\mathbf{S}_{2}\right)$, stated in the introduction.

Let $\Omega$ be a $\Gamma$-invariant bounded smooth domain in $\mathbb{R}^{N}$ which contains the origin. Fix $R>0$ such that $B_{R}(0):=\left\{x \in \mathbb{R}^{N}:|x|<R\right\} \subset \Omega$ and set

$$
\Omega_{\delta}:=\{x \in \Omega:|x|>\delta\} \quad \text { if } \delta \in(0, R), \quad \Omega_{0}:=\Omega .
$$

We consider the problem

$$
\left(\wp_{\delta}^{\phi}\right) \quad \begin{cases}-\Delta u=|u|^{2^{*}-2} u & \text { in } \Omega_{\delta}, \\ u=0 & \text { on } \partial \Omega_{\delta}, \\ u(\gamma x)=\phi(\gamma) u(x) & \forall \gamma \in \Gamma, x \in \Omega_{\delta},\end{cases}
$$

which is the problem (2.2) with $\Theta=\Omega_{\delta}$. We write $J_{\delta}, \mathcal{N}_{\delta}^{\phi}$ and $c_{m, \delta}^{\phi}$ instead of $J_{\Omega_{\delta}}$, $\mathcal{N}_{\Omega_{\delta}}^{\phi}$ and $c_{m, \Omega_{\delta}}^{\phi}$ for its associated energy functional, Nehari manifold and minimax 
values, respectively. Note that $\left(\mathbf{S}_{2}\right)$ implies that $\Omega_{\delta}$ satisfies (2.1) for every $\delta \in$ $[0, R)$.

If $\delta \in(0, R)$ then every $\Gamma$-orbit in $\Omega_{\delta}$ has positive dimension. Hence, Theorem 2.1 asserts that, for each $m \in \mathbb{N}$ and $\delta \in(0, R)$, there exists a nontrivial solution $u_{m, \delta}$ to the problem $\left(\wp_{\delta}^{\phi}\right)$ such that $J_{\delta}\left(u_{m, \delta}\right)=c_{m, \delta}^{\phi}$ and, moreover,

$$
B_{N} m \leq c_{m, \delta_{2}}^{\phi} \leq c_{m, \delta_{1}}^{\phi}<\infty \quad \text { if } 0<\delta_{2} \leq \delta_{1}<R .
$$

We wish to analize the limit profile of $u_{m, \delta}$ as $\delta \rightarrow 0$, for each fixed $m \in \mathbb{N}$. To this end, we consider the limit problem

$$
\left(\wp_{\infty}^{\phi}\right) \quad\left\{\begin{array}{l}
-\Delta u=|u|^{2^{*}-2} u \\
u \in D^{1,2}\left(\mathbb{R}^{N}\right) \\
u(\gamma x)=\phi(\gamma) u(x) \quad \forall \gamma \in \Gamma, x \in \mathbb{R}^{N},
\end{array}\right.
$$

we write $J_{\infty}: D^{1,2}\left(\mathbb{R}^{N}\right)^{\phi} \rightarrow \mathbb{R}$ and $\mathcal{N}_{\infty}^{\phi}$ for the functional and the Nehari manifold associated to it, and we set

$$
c_{\infty}^{\phi}:=\inf _{\mathcal{N}_{\infty}^{\phi}} J_{\infty}(u)
$$

Lemma 3.1. We have that

(a) $c_{1,0}^{\phi}=c_{\infty}^{\phi}$ and $c_{1,0}^{\phi}$ is not attained by $J_{0}$ on $\mathcal{N}_{0}^{\phi}$.

(b) $c_{1, \delta}^{\phi} \rightarrow c_{\infty}^{\phi}$ as $\delta \rightarrow 0$.

Proof. Note that, for every $\delta \in[0, R)$,

$$
c_{1, \delta}^{\phi}=\inf _{u \in \mathcal{N}_{\delta}^{\phi}} J_{\delta}(u)
$$

Then, as $0 \in \Omega$ and 0 is a fixed point of any group action, statement (a) follows from Theorem 2.3 in [5]. To prove (b), let $\eta>0$ and choose $v \in \mathcal{N}_{0}^{\phi}$ such that $J_{0}(v)<c_{1,0}^{\phi}+\eta$. Let $\chi \in \mathcal{C}^{\infty}\left(\mathbb{R}^{N}\right)$ be a radial function such that $\chi(x)=0$ if $|x| \leq 1$ and $\chi(x)=1$ if $|x| \geq 2$, and set $\chi_{\delta}(x):=\chi\left(\frac{x}{\delta}\right)$ and $v_{\delta}:=v \chi_{\delta}$. It is easy to verify that $\left\|v_{\delta}\right\| \rightarrow\|v\|$. Hence, for all sufficiently small $\delta$, there exists $t_{\delta} \in(0, \infty)$ such that $v_{\delta} \in \mathcal{N}_{\delta}^{\phi}$ and

$$
c_{\infty}^{\phi}=c_{1,0}^{\phi} \leq c_{1, \delta}^{\phi} \leq J_{\delta}\left(v_{\delta}\right)<c_{1,0}^{\phi}+2 \eta .
$$

This proves (b).

Next, we will prove the following result.

Theorem 3.2. Assume that the problem $\left(\wp_{0}^{\phi}\right)$ does not have a nontrivial solution, and let $u_{k}$ be a solution to the problem $\left(\wp_{\delta_{k}}^{\phi}\right)$ such that $\delta_{k} \rightarrow 0$ and $J_{\delta_{k}}\left(u_{k}\right) \rightarrow c>0$. Then, after passing to a subsequence, there exist $\ell$ nontrivial solutions $\omega_{1}, \ldots, \omega_{\ell}$ to the problem $\left(\wp_{\infty}^{\phi}\right)$ and $\ell$ sequences of positive numbers $\left(\varepsilon_{j, k}\right), j=1, \ldots, \ell$, with the following properties:

(i) $\varepsilon_{j, k} \rightarrow 0$ and $\frac{\varepsilon_{j, k}}{\delta_{k}} \rightarrow \infty$ as $k \rightarrow \infty$, for each $j=1, \ldots, \ell$; 
(ii) $\left\|u_{k}-\sum_{j=1}^{\ell} \varepsilon_{j, k}^{(2-N) / 2} \omega_{j}\left(\frac{\cdot}{\varepsilon_{j, k}}\right)\right\| \rightarrow 0$ as $k \rightarrow \infty$;

(iii) $c=\sum_{j=1}^{\ell} J_{\infty}\left(\omega_{j}\right)$.

To prove Theorem 3.2, we start with the following definition.

Definition 3.3. A sequence $\left(u_{k}\right)$ such that $u_{k} \in D_{0}^{1,2}\left(\Omega_{\delta_{k}}\right)^{\phi}, \delta_{k} \rightarrow 0, J_{\delta_{k}}\left(u_{k}\right) \rightarrow c$, and $J_{\delta_{k}}^{\prime}\left(u_{k}\right) v_{k} \rightarrow 0$ for every sequence $\left(v_{k}\right)$ with $v_{k} \in D_{0}^{1,2}\left(\Omega_{\delta_{k}}\right)$ and $\left(v_{k}\right)$ bounded in $D^{1,2}\left(\mathbb{R}^{N}\right)$, will be called a concentrating sequence.

As we shall see below, the sequence $\left(u_{k}\right)$ in Theorem 3.2 is a concentrating sequence.

Recall that the $\Gamma$-orbit $\Gamma x$ of a point $x \in \mathbb{R}^{N}$ is $\Gamma$-homeomorphic to the homogeneous space $\Gamma / \Gamma_{x}$, where $\Gamma_{x}:=\{\gamma \in \Gamma: \gamma x=x\}$ is the isotropy group of $x$.

Lemma 3.4. Given sequences $\left(\varepsilon_{k}\right)$ in $(0, \infty)$ and $\left(\xi_{k}\right)$ in $\mathbb{R}^{N}$, there exist a sequence $\left(\zeta_{k}\right)$ in $\mathbb{R}^{N}$ and a closed subgroup $K$ of $\Gamma$ such that, after passing to a subsequence, the following statements hold true:

- The sequence $\left(\varepsilon_{k}^{-1} \operatorname{dist}\left(\Gamma \xi_{k}, \zeta_{k}\right)\right)$ is bounded.

- $\Gamma_{\zeta_{k}}=K$ for all $k \in \mathbb{N}$.

- If $K \neq \Gamma$ then there exists a countable subset $\left\{\gamma_{n}: n \in \mathbb{N}\right\}$ of $\Gamma$ such that

$$
\varepsilon_{k}^{-1}\left|\gamma_{i} \zeta_{k}-\gamma_{j} \zeta_{k}\right| \rightarrow \infty \quad \text { as } k \rightarrow \infty \quad \text { if } i \neq j
$$

Proof. Note that $\left(\mathbf{S}_{1}\right)$ implies that $\Gamma / \Gamma_{x}$ is finite iff $x=0$. So this lemma is just Lemma 3.3 in [7] for this particular situation.

The main step in the proof of Theorem 3.2 is the following proposition.

Proposition 3.5. If $\left(u_{k}\right)$ is a concentrating sequence with $u_{k} \in D_{0}^{1,2}\left(\Omega_{\delta_{k}}\right)^{\phi}$ and $J_{\delta_{k}}\left(u_{k}\right) \rightarrow c>0$, such that $u_{k} \rightarrow 0$ weakly in $D_{0}^{1,2}(\Omega)$, then, after passing to a subsequence, there exist a nontrivial solution $\omega$ to the problem $\left(\wp_{\infty}^{\phi}\right)$, a sequence of positive numbers $\left(\varepsilon_{k}\right)$ and a concentrating sequence $\left(\bar{u}_{k}\right)$, with the following properties:

(a) $\varepsilon_{k} \rightarrow 0$ and $\frac{\varepsilon_{k}}{\delta_{k}} \rightarrow \infty$ as $k \rightarrow \infty$;

(b) $\left\|u_{k}-\bar{u}_{k}-\varepsilon_{k}^{\frac{2-N}{2}} \omega\left(\frac{\cdot}{\varepsilon_{k}}\right)\right\| \rightarrow 0$ as $k \rightarrow \infty$;

(c) $\bar{u}_{k} \in D_{0}^{1,2}\left(\Omega_{\delta_{k}}\right)^{\phi}$ and $J_{\delta_{k}}\left(\bar{u}_{k}\right) \rightarrow c-J_{\infty}(\omega)$ as $k \rightarrow \infty$.

Proof. Since the proof is rather long, we subdivide it into five steps.

STEP 1. We define $\left(\varepsilon_{k}\right)$ and $\omega$. 
Fix $d \in\left(0, \frac{1}{2} S^{N / 2}\right)$. As $\left(u_{k}\right)$ is a concentrating sequence and $c>0$, we have that $\frac{1}{N}\left|u_{k}\right|_{2^{*}}^{2^{*}}=J_{\delta_{k}}\left(u_{k}\right)-\frac{1}{2} J_{\delta_{k}}^{\prime}\left(u_{k}\right) u_{k} \rightarrow c$ and $c \geq \frac{1}{N} S^{N / 2}$. Hence, there are bounded sequences $\left(\varepsilon_{k}\right)$ in $(0, \infty)$ and $\left(\xi_{k}\right)$ in $\mathbb{R}^{N}$ such that, after passing to a subsequence,

$$
\sup _{x \in \mathbb{R}^{N}} \int_{B_{\varepsilon_{k}}(x)}\left|u_{k}\right|^{2^{*}}=\int_{B_{\varepsilon_{k}}\left(\xi_{k}\right)}\left|u_{k}\right|^{2^{*}}=d .
$$

For $\left(\varepsilon_{k}\right)$ and $\left(\xi_{k}\right)$ we choose $K$ and $\left(\zeta_{k}\right)$ as in Lemma 3.4. Then, $\Gamma_{\zeta_{k}}=K$ and $\operatorname{dist}\left(\Gamma \xi_{k}, \zeta_{k}\right)<C \varepsilon_{k}$ for some positive constant $C$ and all $k \in \mathbb{N}$. As $\left|u_{k}\right|$ is $\Gamma$ invariant, we have that

$$
d=\int_{B_{\varepsilon_{k}}\left(\xi_{k}\right)}\left|u_{k}\right|^{2^{*}} \leq \int_{B_{(C+1) \varepsilon_{k}}\left(\zeta_{k}\right)}\left|u_{k}\right|^{2^{*}} .
$$

If $K \neq \Gamma$ then, for each $n \in \mathbb{N}$ and $\gamma_{1}, \ldots, \gamma_{n} \in \Gamma$ as in Lemma 3.4, there exists $k_{n}>0$ such that $\left|\gamma_{i} \zeta_{k}-\gamma_{j} \zeta_{k}\right| \geq 2(C+1) \varepsilon_{k}$ for all $k \geq k_{n}$ and $i \neq j$. Hence,

$$
n d \leq \sum_{j=1}^{n} \int_{B_{(C+1) \varepsilon_{k}}\left(\gamma_{j} \zeta_{k}\right)}\left|u_{k}\right|^{2^{*}} \leq\left|u_{k}\right|_{2^{*}}^{2^{*}} \quad \forall k \geq k_{n} \text { and } \forall n \in \mathbb{N},
$$

which is impossible because $\left(\left|u_{k}\right|_{2^{*}}\right)$ is bounded. Therefore, $K=\Gamma$, and assumption $\left(\mathbf{S}_{1}\right)$ implies that $\zeta_{k}=0$ for all $k \in \mathbb{N}$.

We define

$$
w_{k}(z):=\varepsilon_{k}^{\frac{N-2}{2}} u_{k}\left(\varepsilon_{k} z\right) .
$$

As $\left\|w_{k}\right\|=\left\|u_{k}\right\|$, the sequence $\left(w_{k}\right)$ is bounded in $D^{1,2}\left(\mathbb{R}^{N}\right)$. So, after passing to a subsequence, $w_{k} \rightarrow \omega$ weakly in $D^{1,2}\left(\mathbb{R}^{N}\right), w_{k} \rightarrow \omega$ strongly in $L_{l o c}^{2}\left(\mathbb{R}^{N}\right)$, and $w_{k} \rightarrow \omega$ a.e. in $\mathbb{R}^{N}$. Note that $w_{k} \in D^{1,2}\left(\mathbb{R}^{N}\right)^{\phi}$. Hence, $\omega \in D^{1,2}\left(\mathbb{R}^{N}\right)^{\phi}$.

SteP 2. We show that $\omega \neq 0$.

Arguing by contradiction, assume that $\omega=0$. Let $\varphi \in \mathcal{C}_{c}^{\infty}\left(B_{1}(z)\right)$ for some $z \in$ $\mathbb{R}^{N}$. Observe that, since $\left(u_{k}\right)$ is a concentrating sequence, setting $\varphi_{k}(x):=\varphi\left(\frac{x}{\varepsilon_{k}}\right)$ we have

$$
\begin{aligned}
\int_{\mathbb{R}^{N}} \nabla w_{k} \cdot \nabla\left(\varphi^{2} w_{k}\right)-\int_{\mathbb{R}^{N}} \varphi^{2}\left|w_{k}\right|^{2^{*}} & =\int_{\Omega_{\delta_{k}}} \nabla u_{k} \cdot \nabla\left(\varphi_{k}^{2} u_{k}\right)-\int_{\Omega_{\delta_{k}}} \varphi_{k}^{2}\left|u_{k}\right|^{2^{*}} \\
& =J_{\delta_{k}}^{\prime}\left(u_{k}\right)\left[\varphi_{k}^{2} u_{k}\right]=o(1) .
\end{aligned}
$$

Hence, as $w_{k} \rightarrow \omega$ strongly in $L_{\text {loc }}^{2}\left(\mathbb{R}^{N}\right)$, from (3.1) and our choice of $d$ we obtain

$$
\begin{aligned}
\int_{\mathbb{R}^{N}}\left|\nabla\left(\varphi w_{k}\right)\right|^{2} & =\int_{B_{1}(z)} \varphi^{2}\left|\nabla w_{k}\right|^{2}+\int_{B_{1}(z)} 2 \varphi w_{k} \nabla w_{k} \cdot \nabla \varphi+\int_{B_{1}(z)} w_{k}^{2}|\nabla \varphi|^{2} \\
& =\int_{B_{1}(z)} \nabla w_{k} \cdot \nabla\left(\varphi^{2} w_{k}\right)+o(1)=\int_{B_{1}(z)} \varphi^{2}\left|w_{k}\right|^{2^{*}}+o(1) \\
& \leq\left(\int_{B_{1}(z)}\left|w_{k}\right|^{2^{*}}\right)^{\frac{2^{*}-2}{2^{*}}}\left(\int_{\mathbb{R}^{N}}\left|\varphi w_{k}\right|^{2^{*}}\right)^{\frac{2}{2^{*}}}+o(1) \\
& \leq \frac{d^{2 / N}}{S} \int_{\mathbb{R}^{N}}\left|\nabla\left(\varphi w_{k}\right)\right|^{2}+o(1)<\left(\frac{1}{2}\right)^{2 / N} \int_{\mathbb{R}^{N}}\left|\nabla\left(\varphi w_{k}\right)\right|^{2}+o(1) .
\end{aligned}
$$


Therefore, $\left\|\varphi w_{k}\right\|=o(1)$ and, hence, $\left|\varphi w_{k}\right|_{2^{*}}=o(1)$ for every $\varphi \in \mathcal{C}_{c}^{\infty}\left(B_{1}(z)\right)$, $z \in \mathbb{R}^{N}$. As $B_{C+1}(0) \subset B_{1}\left(z_{1}\right) \cup \cdots \cup B_{1}\left(z_{n}\right)$ for some $z_{1}, \ldots, z_{n} \in \mathbb{R}^{N}$, choosing a smooth partition of unity $\left\{\varphi_{1}^{2^{*}}, \ldots, \varphi_{n}^{2^{*}}\right\}$ with $\operatorname{supp}\left(\varphi_{j}\right) \subset B_{1}\left(z_{j}\right)$, from (3.2) we obtain that

$$
d \leq \int_{B_{C+1}(0)}\left|w_{k}\right|^{2^{*}} \leq \sum_{j=1}^{n} \int_{\mathbb{R}^{N}}\left|\varphi_{j} w_{k}\right|^{2^{*}}=o(1) .
$$

This is a contradiction. Consequently, $\omega \neq 0$.

STEP 3. We prove (a) and we show that $\omega$ solves the problem $\left(\wp_{\infty}^{\phi}\right)$.

As $\left(\varepsilon_{k}\right)$ is bounded, after passing to a subsequence, we have that $\varepsilon_{k} \rightarrow \varepsilon \in[0, \infty)$. Since $u_{k} \rightarrow 0$ weakly in $D_{0}^{1,2}(\Omega)$ and $w \neq 0$, an easy argument shows that $\varepsilon=0$.

Inequality (3.2) implies that $(C+1) \varepsilon_{k}>\delta_{k}$ for all $k \in \mathbb{N}$. Therefore, if $\left(\frac{\varepsilon_{k}}{\delta_{k}}\right)$ is bounded, then, after passing to a subsequence, $\frac{\varepsilon_{k}}{\delta_{k}} \rightarrow \varrho \in(0, \infty)$. As $w_{k}=0$ in $B_{\delta_{k} / \varepsilon_{k}}(0)$ and $w_{k} \rightarrow \omega$ a.e. in $\mathbb{R}^{N}$, we have that $\omega=0$ a.e. in $B_{1 / \varrho}(0)$. Moreover, for every $\varphi \in \mathcal{C}_{c}^{\infty}\left(\mathbb{R}^{N} \backslash \overline{B_{1 / \varrho}(0)}\right)$, setting $\varphi_{k}(x):=\varphi\left(\frac{x}{\varepsilon_{k}}\right)$ we have that $\operatorname{supp}\left(\varphi_{k}\right) \subset \Omega_{\delta_{k}}$ for $k$ large enough and, as $\left(u_{k}\right)$ is a concentrating sequence, we obtain

$$
\begin{aligned}
\int_{\mathbb{R}^{N}} \nabla w_{k} \cdot \nabla \varphi-\int_{\mathbb{R}^{N}}\left|w_{k}\right|^{2^{*}-2} w_{k} \varphi & =\int_{\Omega_{\delta_{k}}} \nabla u_{k} \cdot \nabla \varphi_{k}-\int_{\Omega_{\delta_{k}}}\left|u_{k}\right|^{2^{*}-2} u_{k} \varphi_{k} \\
& =J_{\delta_{k}}^{\prime}\left(u_{k}\right)\left[\varphi_{k}\right]=o(1) .
\end{aligned}
$$

Since $w_{k} \rightarrow \omega$ weakly in $D^{1,2}\left(\mathbb{R}^{N}\right)$, this implies that $J_{\infty}^{\prime}(\omega) \varphi=0$ for every $\varphi \in$ $\mathcal{C}_{c}^{\infty}\left(\mathbb{R}^{N} \backslash \overline{B_{1 / \varrho}(0)}\right)$, i.e., $\omega$ is a nontrivial solution to the problem

$$
-\Delta u=|u|^{2^{*}-2} u, \quad u \in D_{0}^{1,2}\left(\mathbb{R}^{N} \backslash B_{1 / \varrho}(0)\right),
$$

which is impossible; see Remark 4.1 below.

Therefore, $\left(\frac{\varepsilon_{k}}{\delta_{k}}\right)$ is unbounded. So, after passing to a subsequence, $\frac{\varepsilon_{k}}{\delta_{k}} \rightarrow \infty$ and a similar argument shows that $J_{\infty}^{\prime}(\omega) \varphi=0$ for every $\varphi \in \mathcal{C}_{c}^{\infty}\left(\mathbb{R}^{N} \backslash\{0\}\right)$. Since $\mathcal{C}_{c}^{\infty}\left(\mathbb{R}^{N} \backslash\{0\}\right)$ is dense in $D^{1,2}\left(\mathbb{R}^{N}\right)$, we conclude that $\omega$ is a solution to the problem $\left(\wp_{\infty}^{\phi}\right)$.

SteP 4. We define $\left(\bar{u}_{k}\right)$ and we prove $(\mathrm{b})$.

Let $\chi \in \mathcal{C}_{c}^{\infty}\left(\mathbb{R}^{N}\right)$ be a radial function such that $0 \leq \chi(z) \leq 1, \chi(z)=1$ if $\frac{1}{2} \leq|z| \leq 2$, and $\chi(z)=0$ if $|z| \leq \frac{1}{4}$ or $|z| \geq 4$. Let $R>0$ be such that $B_{R}(0) \subset \Omega$ and, for $\delta_{k} \in\left(0, \frac{R}{16}\right)$, set

$$
\chi_{k}(z):=\left\{\begin{array}{cl}
\chi\left(\frac{\varepsilon_{k}}{4 \delta_{k}} z\right) & \text { if } 0 \leq|z| \leq \frac{4 \delta_{k}}{\varepsilon_{k}}, \\
1 & \text { if } \frac{4 \delta_{k}}{\varepsilon_{k}} \leq|z| \leq \frac{R}{4 \varepsilon_{k}}, \\
\chi\left(\frac{4 \varepsilon_{k}}{R} z\right) & \text { if } \frac{R}{4 \varepsilon_{k}} \leq|z|,
\end{array}\right.
$$

and $\widehat{\chi}_{k}(x):=\chi_{k}\left(\frac{x}{\varepsilon_{k}}\right)$. Note that $\operatorname{supp}\left(\widehat{\chi}_{k}\right) \subset\left\{x \in \mathbb{R}^{N}: \delta_{k} \leq|x| \leq R\right\}$. We define

$$
\bar{u}_{k}(x):=u_{k}(x)-\varepsilon_{k}^{\frac{2-N}{2}} \omega\left(\frac{x}{\varepsilon_{k}}\right) \widehat{\chi}_{k}(x) .
$$


Then, $\bar{u}_{k} \in D_{0}^{1,2}\left(\Omega_{\delta_{k}}\right)^{\phi}$. A straightforward computation shows that

$$
\lim _{k \rightarrow \infty}\left\|\chi_{k} w-w\right\|=0 \quad \forall w \in D^{1,2}\left(\mathbb{R}^{N}\right) .
$$

So, performing the change of variable $x=\varepsilon_{k} z$, we obtain

$$
\left\|u_{k}-\bar{u}_{k}-\varepsilon_{k}^{\frac{2-N}{2}} \omega\left(\frac{x}{\varepsilon_{k}}\right)\right\|^{2}=\left\|\chi_{k} \omega-\omega\right\|^{2}=o(1) .
$$

This proves (b).

SteP 5. We prove (c) and we show that $\left(\bar{u}_{k}\right)$ is a concentrating sequence.

As $w_{k} \rightarrow \omega$ weakly in $D^{1,2}\left(\mathbb{R}^{N}\right)$, with the change of variable $x=\varepsilon_{k} z$ and using (3.3), we obtain

$$
\left\|u_{k}\right\|^{2}-\left\|\bar{u}_{k}\right\|^{2}=\left\|w_{k}\right\|^{2}-\left\|w_{k}-\chi_{k} \omega\right\|^{2}=\|\omega\|^{2}+o(1) .
$$

Using the Brezis-Lieb lemma, we obtain in a similar way

$$
\left|u_{k}\right|_{2^{*}}^{2^{*}}-\left|\bar{u}_{k}\right|_{2^{*}}^{2^{*}}=\left|w_{k}\right|_{2^{*}}^{2^{*}}-\left|w_{k}-\chi_{k} \omega\right|_{2^{*}}^{2^{*}}=|\omega|_{2^{*}}^{2^{*}}+o(1) .
$$

It follows that

$$
J_{\delta_{k}}\left(\bar{u}_{k}\right)=J_{\delta_{k}}\left(u_{k}\right)-J_{\infty}(\omega)+o(1)=c-J_{\infty}(\omega)+o(1) .
$$

This proves $(\mathrm{c})$. To prove that $\left(\bar{u}_{k}\right)$ is a concentrating sequence, we first consider the function $f(r):=|r|^{2^{*}-2} r$. Given $r, s \in \mathbb{R}$, the mean value theorem yields a number $t \in(0,1)$ such that

$$
|f(s)-f(s-r)| \leq C|s-t r|^{2^{*}-2}|r| \leq C\left(|s|^{2^{*}-2}+|r|^{2^{*}-2}\right)|r| .
$$

Setting $s:=w_{k}(z)-\omega(z)$ and $s-r:=w_{k}(z)-\chi_{k}(z) \omega(z)$, for any $\varphi \in \mathcal{C}_{c}^{\infty}\left(\mathbb{R}^{N}\right)$ we obtain

$$
\begin{gathered}
\int_{\mathbb{R}^{N}}|| w_{k}-\left.\omega\right|^{2^{*}-2}\left(w_{k}-\omega\right) \varphi-\left|w_{k}-\chi_{k} \omega\right|^{2^{*}-2}\left(w_{k}-\chi_{k} \omega\right) \varphi \mid \\
\leq C \int_{\mathbb{R}^{N}}\left(\left|w_{k}-\omega\right|^{2^{*}-2}+\left|\chi_{k} \omega-\omega\right|^{2^{*}-2}\right)\left|\chi_{k} \omega-\omega\right||\varphi| \\
\leq C\left(\left|w_{k}-\omega\right|_{2^{*}}^{2^{*}-2}+|\omega|_{2^{*}}^{2^{*}-2}\right)\left\|\chi_{k} \omega-\omega\right\|\|\varphi\|=o(1)\|\varphi\| .
\end{gathered}
$$

This estimate, together with Lemma 8.9 in [22], yields

$$
\left|w_{k}\right|^{2^{*}-2} w_{k}-\left|w_{k}-\chi_{k} \omega\right|^{2^{*}-2}\left(w_{k}-\chi_{k} \omega\right)-|\omega|^{2^{*}-2} \omega \longrightarrow 0 \text { in }\left(D^{1,2}\left(\mathbb{R}^{N}\right)\right)^{\prime}
$$

Let $v_{k} \in D_{0}^{1,2}\left(\Omega_{\delta_{k}}\right)$ be such that $\left(v_{k}\right)$ is bounded in $D^{1,2}\left(\mathbb{R}^{N}\right)$, and set $\widetilde{v}_{k}(z):=$ $\varepsilon_{k}^{\frac{N-2}{2}} v_{k}\left(\varepsilon_{k} z\right)$. As $J_{\infty}^{\prime}(\omega)=0$, with the usual change of variable and using identities (3.3) and (3.4), we obtain

$$
\begin{gathered}
\left|J_{\delta_{k}}^{\prime}\left(u_{k}\right) v_{k}-J_{\delta_{k}}^{\prime}\left(\bar{u}_{k}\right) v_{k}\right|=\left|J_{\infty}^{\prime}\left(w_{k}\right) \widetilde{v}_{k}-J_{\infty}^{\prime}\left(w_{k}-\chi_{k} \omega\right) \widetilde{v}_{k}-J_{\infty}^{\prime}(\omega) \widetilde{v}_{k}\right| \\
\leq\left\|J_{\infty}^{\prime}\left(w_{k}\right)-J_{\infty}^{\prime}\left(w_{k}-\chi_{k} \omega\right)-J_{\infty}^{\prime}(\omega)\right\|_{\left(D^{1,2}\left(\mathbb{R}^{N}\right)\right)^{\prime}}\left\|\widetilde{v}_{k}\right\|=o(1) .
\end{gathered}
$$

Since $J_{\delta_{k}}^{\prime}\left(u_{k}\right) v_{k} \rightarrow 0$, we conclude that $J_{\delta_{k}}^{\prime}\left(\bar{u}_{k}\right) v_{k} \rightarrow 0$. This finishes the proof. 
Proof of Theorem 3.2. Let $u_{k}$ be a solution to the problem $\left(\wp_{\delta_{k}}^{\phi}\right)$ such that $\delta_{k} \rightarrow 0$ and $J_{\delta_{k}}\left(u_{k}\right) \rightarrow c>0$. Note that, by the principle of symmetric criticality [18], $u_{k}$ is a critical point of the functional $J_{\delta_{k}}$ in the whole space $D_{0}^{1,2}\left(\Omega_{\delta_{k}}\right)$, i.e., $J_{\delta_{k}}^{\prime}\left(u_{k}\right) v=0$ for every $v \in D_{0}^{1,2}\left(\Omega_{\delta_{k}}\right)$. So, $\left(u_{k}\right)$ is a concentrating sequence. Since $J_{\delta_{k}}\left(u_{k}\right)=$ $\frac{1}{N}\left\|u_{k}\right\|^{2}$, the sequence $\left(u_{k}\right)$ is bounded and, after passing to a subsequence, $u_{k} \rightarrow u$ weakly in $D_{0}^{1,2}(\Omega)$. Now, if $\varphi \in \mathcal{C}_{c}^{\infty}(\Omega \backslash\{0\})$, then $\operatorname{supp}(\varphi) \subset \Omega_{\delta_{k}}$ for $k$ large enough. Therefore,

$$
J_{0}^{\prime}\left(u_{k}\right) \varphi=\int_{\Omega_{\delta_{k}}} \nabla u_{k} \cdot \nabla \varphi-\int_{\Omega_{\delta_{k}}}\left|u_{k}\right|^{2^{*}-2} u_{k} \varphi=J_{\delta_{k}}^{\prime}\left(u_{k}\right) \varphi=0,
$$

for $k$ large enough. Passing to the limit, we get that $J_{0}^{\prime}(u) \varphi=0$ for every $\varphi \in$ $\mathcal{C}_{c}^{\infty}(\Omega \backslash\{0\})$ and, as $\mathcal{C}_{c}^{\infty}(\Omega \backslash\{0\})$ is dense in $D_{0}^{1,2}(\Omega)$, we conclude that $u$ is a solution to the problem $\left(\wp_{0}^{\phi}\right)$. Thus, by assumption, $u=0$.

Now we can apply Proposition 3.5 to $\left(u_{k}\right)$ to obtain a new concentrating sequence $\left(u_{k}^{1}\right)$. Then, we apply it to $\left(u_{k}^{1}\right)$, so on. It is easy to see that concentrating sequences occur only at levels $c \geq 0$. So, after a finite number of steps, we will arrive to a concentrating sequence $\left(u_{k}^{\ell}\right)$ such that $J_{\delta_{k}}\left(u_{k}^{\ell}\right) \rightarrow 0$. This finishes the process, and yields the result.

Proof of Theorem 1.1. By Theorem 2.1, there exists a nontrivial solution $u_{m, \delta}$ to the problem $\left(\wp_{\delta}^{\phi}\right)$ such that $J_{\delta}\left(u_{m, \delta}\right)=\frac{1}{N}\left\|u_{m, \delta}\right\|^{2}=c_{m, \delta}^{\phi}$ and

$$
B_{N} m \leq c_{m, \delta}^{\phi} \quad \text { for every } \delta \in(0, R), m \in \mathbb{N} .
$$

If $\Omega$ is strictly starshaped then the problem $\left(\wp_{0}^{\phi}\right)$ does not have a nontrivial solution, and the second statement in Theorem 1.1 follows immediately from Theorem 3.2.

\section{TOWERS OF NODAL BUBBLES FOR ANNULI}

In this section we analize the case when $\Omega$ is a ball, i.e., $\Omega:=\left\{x \in \mathbb{R}^{N}:|x|<R\right\}$. We will show that, for every $\ell \in \mathbb{N}$, one can construct solutions whose limit profile is a tower of $\ell \phi$-nodal bubbles. To do this, we will take advantage of the geometry of the problem.

As before, we will assume that $\Gamma$ is a closed subgroup of $O(N)$ and $\phi: \Gamma \rightarrow \mathbb{Z}_{2}$ is a continuous homomorphism, which satisfy $\left(\mathbf{S}_{1}\right)$ and $\left(\mathbf{S}_{2}\right)$.

For each $s>0$, we consider the inversion on the sphere $S_{s}:=\left\{x \in \mathbb{R}^{N}:|x|=s\right\}$, given by

$$
\mathfrak{K}_{s}(x):=\frac{s^{2} x}{|x|^{2}} \quad \text { for } \quad x \neq 0 .
$$

We define the image under $\mathfrak{K}_{s}$ of a domain $\Theta$ in $\mathbb{R}^{N}$ to be the set

$$
\mathfrak{K}_{s}(\Theta):=\left\{\mathfrak{K}_{s}(x): x \in \Theta \backslash\{0\}\right\}
$$


and, for $u: \mathfrak{K}_{s}(\Theta) \rightarrow \mathbb{R}$, we consider its Kelvin transform, which is the function $\mathfrak{K}_{s} u: \Theta \rightarrow \mathbb{R}$ defined by

$$
\left(\mathfrak{K}_{s} u\right)(x):=\frac{s^{N-2}}{|x|^{N-2}} u\left(\frac{s^{2} x}{|x|^{2}}\right)
$$

It follows easily that

$$
\int_{\Theta} \nabla\left(\mathfrak{K}_{s} u\right) \cdot \nabla\left(\mathfrak{K}_{s} v\right)=\int_{\mathfrak{K}_{s}(\Theta)} \nabla u \cdot \nabla v, \quad \int_{\Theta}\left|\left(\mathfrak{K}_{s} u\right)\right|^{2^{*}}=\int_{\mathfrak{K}_{s}(\Theta)}|u|^{2^{*}},
$$

for every $u, v \in D_{0}^{1,2}\left(\mathfrak{K}_{s}(\Theta)\right)$. Therefore, $u$ solves $-\Delta u=|u|^{2^{*}-2} u$ in $D_{0}^{1,2}\left(\mathfrak{K}_{s}(\Theta)\right)$ if and only if $\mathfrak{K}_{s} u$ solves the same equation in $D_{0}^{1,2}(\Theta)$.

Remark 4.1. Note that this implies, in particular, that the problem

$$
-\Delta u=|u|^{2^{*}-2} u, \quad u \in D_{0}^{1,2}\left(\mathbb{R}^{N} \backslash B_{s}(0)\right),
$$

does not have a nontrivial solution. Otherwise,

$$
-\Delta u=|u|^{2^{*}-2} u, \quad u \in D_{0}^{1,2}\left(B_{s}(0)\right)
$$

would have a nontrivial solution, which is impossible, due to Pohozhaev's identity.

For $w \in D^{1,2}\left(\mathbb{R}^{N}\right)$ and $\varepsilon \in(0, \infty)$, set $w_{\varepsilon}(x):=\varepsilon^{(2-N) / 2} w\left(\frac{x}{\varepsilon}\right)$. It is straightforward to verify that

$$
\mathfrak{K}_{s}^{2} w=w \quad \text { and } \quad \mathfrak{K}_{s}\left(w_{\varepsilon}\right)=\left(\mathfrak{K}_{1} w\right)_{s^{2} / \varepsilon} \quad \text { for every } s, \varepsilon \in(0, \infty) .
$$

Clearly, $\mathfrak{K}_{s}(\gamma x)=\gamma \mathfrak{K}_{s}(x)$ for every $\gamma \in O(N)$. Therefore, $\Theta$ is $\Gamma$-invariant if and only if $\mathfrak{K}_{s}(\Theta)$ is $\Gamma$-invariant, and a function $u: \mathfrak{K}_{s}(\Theta) \rightarrow \mathbb{R}$ is $\phi$-equivariant if and only if $\mathfrak{K}_{s} u: \Theta \rightarrow \mathbb{R}$ is $\phi$-equivariant. In particular, if $\Theta=\mathbb{R}^{N}$, we have that $\omega$ solves the problem $\left(\wp_{\infty}^{\phi}\right)$ iff $\mathfrak{K}_{s} \omega$ solves the problem $\left(\wp_{\infty}^{\phi}\right)$.

For $r, s \in(0, R], r<s$, we consider the annulus $A_{r, s}:=\left\{x \in \mathbb{R}^{N}: r<|x|<s\right\}$. We denote the problem $(2.2)$ in $\Theta=A_{r, s}$ by $\left(\wp_{r, s}^{\phi}\right)$, and we write $J_{r, s}$ and $\mathcal{N}_{r, s}^{\phi}$, instead of $J_{A_{r, s}}$ and $\mathcal{N}_{A_{r, s}}^{\phi}$, for its energy functional and its Nehari manifold. Let

$$
c_{r, s}^{\phi}:=\inf _{\mathcal{N}_{r, s}^{\phi}} J_{r, s}(u)
$$

By Theorem 2.1 there exists a solution $u_{r}$ to the problem $\left(\wp_{r, R}^{\phi}\right)$ such that $J_{r, R}\left(u_{r}\right)=$ $c_{r, R}^{\phi}$. Set $\varrho:=\frac{r}{R}$. Then, as $\mathfrak{K}_{r}\left(A_{r, R}\right)=A_{\varrho r, r}$, we have that $-\mathfrak{K}_{r} u_{r}$ is a solution to the problem $\left(\wp_{\varrho}^{\phi}, r\right)$. A straightforward computation shows that the normal derivatives of $-\mathfrak{K}_{r} u_{r}$ and $u_{r}$ coincide at each point of the common boundary $S_{r}$ of $A_{\varrho r, r}$ and $A_{r, R}$. Therefore, for each $\ell \in \mathbb{N}$, setting

$$
u_{1, r}:=u_{r} \quad \text { and } \quad u_{j, r}:=\mathfrak{K}_{\varrho^{j-2} r} u_{j-1, r} \text { for } j=2, \ldots, \ell,
$$

we have that the function $\widehat{u}_{\ell, r}$ defined by

$$
\widehat{u}_{\ell, r}:=\sum_{j=1}^{\ell}(-1)^{j} u_{j, r}
$$


is a solution to the problem $\left(\wp_{\varrho^{\ell-1} r, R}^{\phi}\right)$ in the annulus $A_{\varrho^{\ell-1} r, R}$ which satisfies

$$
J_{\varrho^{\ell-1} r, R}\left(\widehat{u}_{\ell, r}\right)=\ell c_{r, R}^{\phi} .
$$

The following theorem describes the limit profile of $\widehat{u}_{\ell, r}$ as $r \rightarrow 0$.

Theorem 4.2. Let $u_{r}$ be a solution to the problem $\left(\wp_{r, R}^{\phi}\right)$ with $J_{r, R}\left(u_{r}\right)=c_{r, R}^{\phi}$. Then, there exist $r_{k} \in(0, R)$ with $r_{k} \rightarrow 0$, a nontrivial solution $\omega$ to the problem $\left(\wp_{\infty}^{\phi}\right)$ with $J_{\infty}(\omega)=c_{\infty}^{\phi}$, and a sequence $\left(\varepsilon_{k}\right)$ in $(0, \infty)$ such that the following statements hold true for each $\ell \in \mathbb{N}$ :

(i) $\varepsilon_{k} \rightarrow 0$ and $\frac{\varepsilon_{k}}{r_{k}} \rightarrow 0$ as $k \rightarrow \infty$;

(ii) $\left\|\widehat{u}_{\ell, r_{k}}-\sum_{j=1}^{\ell}(-1)^{j} \varepsilon_{j, k}^{(2-N) / 2} \omega_{j}\left(\frac{\cdot}{\varepsilon_{j, k}}\right)\right\| \rightarrow 0$ as $k \rightarrow \infty$, where $\omega_{j}:=\omega$ and $\varepsilon_{j, k}:=\frac{r_{k}^{j-1} \varepsilon_{k}}{R^{j-1}}$ if $j$ is odd, and $\omega_{j}:=\mathfrak{K}_{1} \omega$ and $\varepsilon_{j, k}:=\frac{r_{k}^{j}}{R^{j-2} \varepsilon_{k}}$ if $j$ is even;

(iii) $J_{r_{k}^{\ell} / R^{\ell-1}, R}\left(\widehat{u}_{\ell, r_{k}}\right) \rightarrow \ell c_{\infty}^{\phi}$ as $k \rightarrow \infty$.

Proof. By Lemma 3.1, we have that $c_{r, R}^{\phi} \rightarrow c_{\infty}^{\phi}$ as $r \rightarrow 0$. Therefore, Theorem 3.2 yields a sequence $r_{k} \in(0, R)$ with $r_{k} \rightarrow 0$, a nontrivial solution $\omega$ to the problem $\left(\wp_{\infty}^{\phi}\right)$ with $J_{\infty}(\omega)=c_{\infty}^{\phi}$, and a sequence $\left(\varepsilon_{k}\right)$ in $(0, \infty)$ such that $\varepsilon_{k} \rightarrow 0, \frac{\varepsilon_{k}}{r_{k}} \rightarrow \infty$ and

$$
\left\|u_{r_{k}}-\varepsilon_{k}^{\frac{2-N}{2}} \omega\left(\frac{\cdot}{\varepsilon_{k}}\right)\right\| \rightarrow 0 \quad \text { as } k \rightarrow \infty
$$

Set $\varrho_{k}:=\frac{r_{k}}{R}$

$$
\bar{\omega}_{1, k}:=\omega_{\varepsilon_{k}} \quad \text { and } \quad \bar{\omega}_{j, k}:=\mathfrak{K}_{\varrho_{k}^{j-2} r_{k}} \bar{\omega}_{j-1, k} \quad \text { for } j=2, \ldots, \ell .
$$

Then, (4.1) yields

$$
\left\|u_{j, r_{k},}-\bar{\omega}_{j, k}\right\|=\left\|\mathfrak{K}_{\varrho_{k}^{j-2} r_{k}} u_{j-1, r_{k}},-\mathfrak{K}_{\varrho_{k}^{j-2} r_{k}} \bar{\omega}_{j-1, k}\right\|=\left\|u_{j-1, r_{k},}-\bar{\omega}_{j-1, k}\right\|
$$

for every $j=2, \ldots, \ell$, and from (4.4) we obtain

$$
\begin{aligned}
\left\|\widehat{u}_{\ell, r_{k}}-\sum_{j=1}^{\ell}(-1)^{j} \bar{\omega}_{j, k}\right\| & =\left\|\sum_{j=1}^{\ell}(-1)^{j}\left(u_{j, r_{k}},-\bar{\omega}_{j, k}\right)\right\| \\
& \leq \sum_{j=1}^{\ell}\left\|u_{j, r_{k}},-\bar{\omega}_{j, k}\right\|=\ell\left\|u_{r_{k}}-\omega_{\varepsilon_{k}}\right\| \longrightarrow 0 \quad \text { as } k \rightarrow \infty .
\end{aligned}
$$

Using the formulas (4.2) we see that

$$
\bar{\omega}_{j, k}=\left\{\begin{array}{lll}
\omega_{\varepsilon_{j, k}} & \text { with } \varepsilon_{j, k}:=\varrho_{k}^{j-1} \varepsilon_{k} & \text { if } j \text { is odd } \\
\left(\mathfrak{K}_{1} \omega\right)_{\varepsilon_{j, k}} & \text { with } \varepsilon_{j, k}:=\varrho_{k}^{j-2} \frac{r_{k}^{2}}{\varepsilon_{k}} & \text { if } j \text { is even. }
\end{array}\right.
$$

This proves (ii). From (4.3) and Lemma 3.1 we immediately obtain (iii). 


\section{REFERENCES}

[1] Ackermann, Nils; Cano, Alfredo; Hernández-Martínez, Eric: Spectral density estimates with partial symmetries and an application to Bahri-Lions-type results. Calc. Var. Partial Differential Equations 56 (2017), no. 1, 56:6.

[2] Bahri, A.; Lions, P.-L.: Morse index of some min-max critical points. I. Application to multiplicity results. Comm. Pure Appl. Math. 41 (1988), no. 8, 1027-1037.

[3] Bracho, Javier; Clapp, Mónica; Marzantowicz, Wacław: Symmetry breaking solutions of nonlinear elliptic systems. Topol. Methods Nonlinear Anal. 26 (2005), no. 1, 189-201.

[4] Chang, Kung-ching: Infinite-dimensional Morse theory and multiple solution problems. Progress in Nonlinear Differential Equations and their Applications, 6. Birkhäuser Boston, Inc., Boston, MA, 1993.

[5] Clapp, Mónica: Entire nodal solutions to the pure critical exponent problem arising from concentration. J. Differential Equations 261 (2016), no. 6, 3042-3060.

[6] Clapp, Mónica; Faya, Jorge: Multiple solutions to the Bahri-Coron problem in some domains with nontrivial topology. Proc. Amer. Math. Soc. 141 (2013), no. 12, 4339-4344.

[7] Clapp, Mónica; Faya, Jorge: Multiple solutions to anisotropic critical and supercritical problems in symmetric domains. Progr. Nonlinear Differential Equations Appl. 86 (2015), 99-120.

[8] Clapp, Mónica; Musso, Monica; Pistoia, Angela: Multipeak solutions to the Bahri-Coron problem in domains with a shrinking hole. J. Funct. Anal. 256 (2009), no. 2, 275-306.

[9] Clapp, Mónica; Pacella, Filomena: Multiple solutions to the pure critical exponent problem in domains with a hole of arbitrary size. Math. Z. 259 (2008), no. 3, 575-589.

[10] Clapp, Mónica; Pacella, Filomena: Existence and asymptotic profile of nodal solutions to supercritical problems, Advanced Nonlinear Studies, in press. DOI:10.1515/ans-2016-6009.

[11] Clapp, Mónica; Weth, Tobias: Two solutions of the Bahri-Coron problem in punctured domains via the fixed point transfer. Commun. Contemp. Math. 10 (2008), no. 1, 81-101.

[12] Coron, Jean-Michel: Topologie et cas limite des injections de Sobolev. C. R. Acad. Sci. Paris Sér. I Math. 299 (1984), no. 7, 209-212.

[13] Cwikel, Michael: Weak type estimates for singular values and the number of bound states of Schrödinger operators. Ann. of Math. (2) 106 (1977), no. 1, 93-100.

[14] Ding, Weiyue: On a conformally invariant elliptic equation on $\mathbb{R}^{n}$. Comm. Math. Phys. 107 (1986), no. 2, 331-335.

[15] Ge, Yuxin; Musso, Monica; Pistoia, Angela: Sign changing tower of bubbles for an elliptic problem at the critical exponent in pierced non-symmetric domains. Comm. Partial Differential Equations 35 (2010), no. 8, 1419-1457.

[16] Lieb, Elliott: Bounds on the eigenvalues of the Laplace and Schroedinger operators. Bull. Amer. Math. Soc. 82 (1976), no. 5, 751-753.

[17] Marino, A.; Prodi, G.: Metodi perturbativi nella teoria di Morse. Boll. Un. Mat. Ital. (4) 11 (1975), no. 3, suppl., 1-32.

[18] Palais, Richard S.: The principle of symmetric criticality. Comm. Math. Phys. 69 (1979), no. $1,19-30$.

[19] Rosenbljum, G.: The distribution of the discrete spectrum for singular differential operators. Soviet Math. Dokl. 13 (1972), 245-249.

[20] Struwe, Michael: A global compactness result for elliptic boundary value problems involving limiting nonlinearities. Math. Z. 187 (1984), no. 4, 511-517.

[21] Struwe, Michael: Variational methods. Applications to nonlinear partial differential equations and Hamiltonian systems. Second edition. Ergebnisse der Mathematik und ihrer Grenzgebiete (3), 34. Springer-Verlag, Berlin, 1996.

[22] Willem, Michel: Minimax theorems. Progress in Nonlinear Differential Equations and their Applications, 24. Birkhäuser Boston, Inc., Boston, MA, 1996 
Instituto de Matemáticas, Universidad Nacional Autónoma de México, Circuito ExTerior, C.U., 04510 Ciudad de México, Mexico

E-mail address: monica.clapp@im.unam.mx

Centro de Modelamiento Matemático, Universidad de Chile, Blanco Encalada 2120, Santiago, Chile

E-mail address: jorgefaya@gmail.com

Dipartimento di Matematica, Università "La Sapienza" di Roma, P.le. Aldo Moro 2, 00185 Roma, Italy.

E-mail address: pacella@mat.uniroma1.it 